\title{
Tumor del seno endodermal extragonadal en la infancia. Reporte de dos casos.
}

\author{
SÁNCHEZ LIHON Juvenal *, CASAVILCA ZAMBRANO Sandro **, GARCIA MADRID Jorge ***, KLINGE \\ CASTRO German ***.
}

\begin{abstract}
SUMMARY
We report two cases of extragonadal pelvic endodermal sinus tumor diagnosed at the Departament of Pediatric of the National Institute of Neoplastic Diseases and discuss the clinical presentation, differential diagnosis and pathologic study. ( Rev Med Hered 2003; 14:98-103 ).
\end{abstract}

KEY WORDS: Extragonadal endodermal sinus tumor.

\section{INTRODUCCION.}

Los tumores de células germinales son neoplasias derivadas de las células germinales primordiales que pueden ser de localización gonadal o extragonadal. El tumor del seno endodermal es el tumor maligno de células germinales más frecuente en la edad pediátrica, cuya localización más frecuente es la región sacrococcigea en recién nacidos (1). En niñas mayores y adolescentes su principal localización es el ovario, con menos frecuencia se localiza en mediastino, retroperitoneo, área pineal y vagina (2). La alfa fetoproteina es la principal proteína sérica del feto, producida en un principio por el saco embrionario, por lo que es un indicador para el diagnóstico, seguimiento y detección de recurrencias (3).

Las niñas con tumor del seno endodermal se presentan generalmente con descenso sanguinolento o una masa que protruye por la vagina $(3,4)$, pudiendo ser tratados exitosamente con cirugía y quimioterapia (5).

CASO I.

Paciente de once meses de edad, natural y procedente de La Libertad, que ingresa al INEN el 14.06.02, referida del Hospital Regional de Trujillo con Diagnóstico por biopsia de neoplasia maligna consistente con carcinoma de células claras. La madre refiere que desde los 6 meses de edad presenta sangrado vaginal escaso que progresivamente se ha incrementado en cantidad y frecuencia.

Examenes auxiliares: $\mathrm{Hb}$ (103), Hto (32.1), AFP $35595.0 \mathrm{ng} / \mathrm{ml}$. Los demás exámenes se encuentran dentro de límites normales. Ecografía: Proceso neoformativo de localización retrovesical que mide 6.5 $\mathrm{cm}$. de diámetro que podría ser de origen uterino o de

* Jefe del Departamento de Patología del INEN.

** Médico Patólogo, Residente INEN de Patología Oncológica.

*** Médico Asistente del Departamento de Patología. 


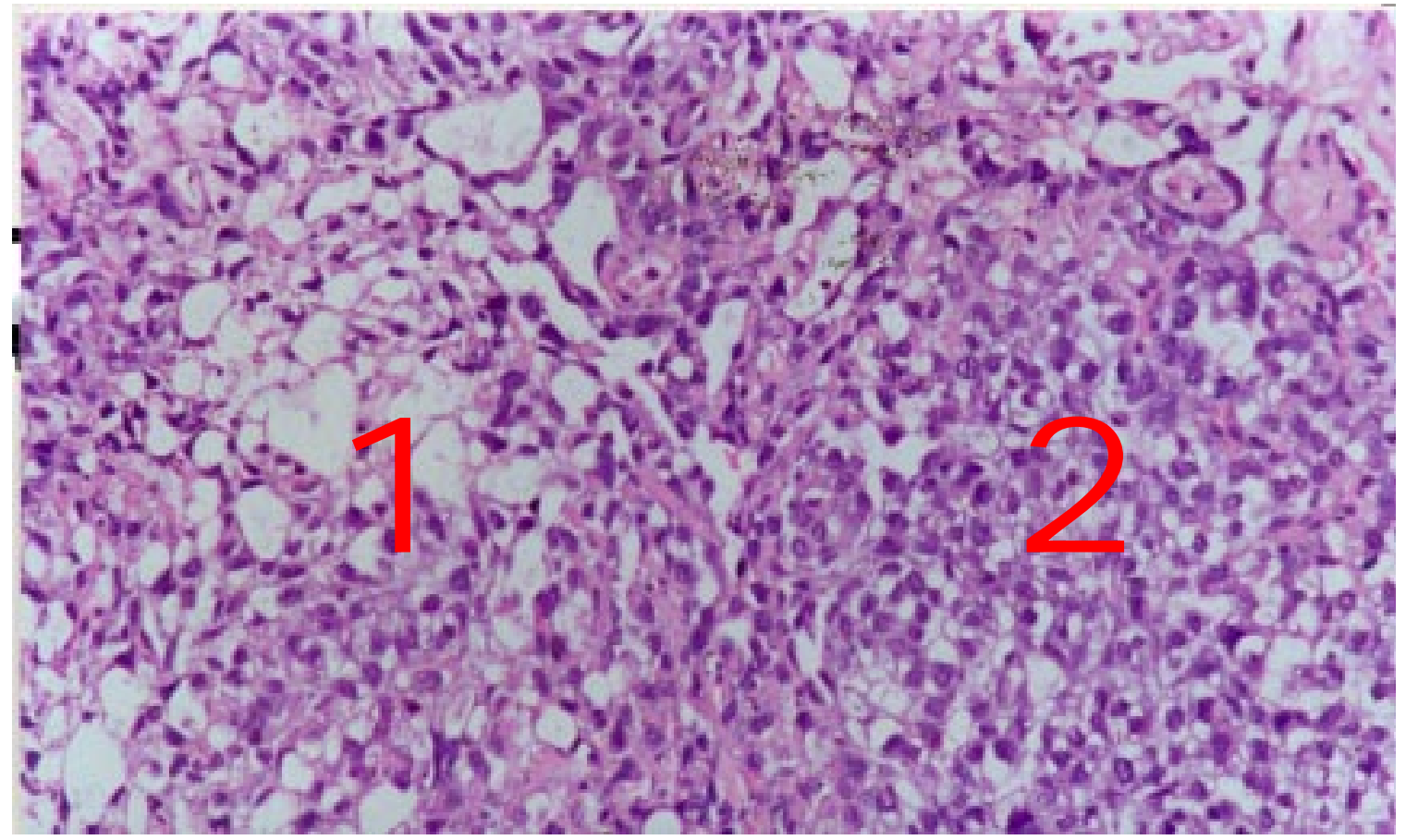

Foto 1. Tumor del seno endodermal en 100 aumentos. Se evidencian patrones histológicos reticular microquistico (1) y sólido (2). H\&E x 100

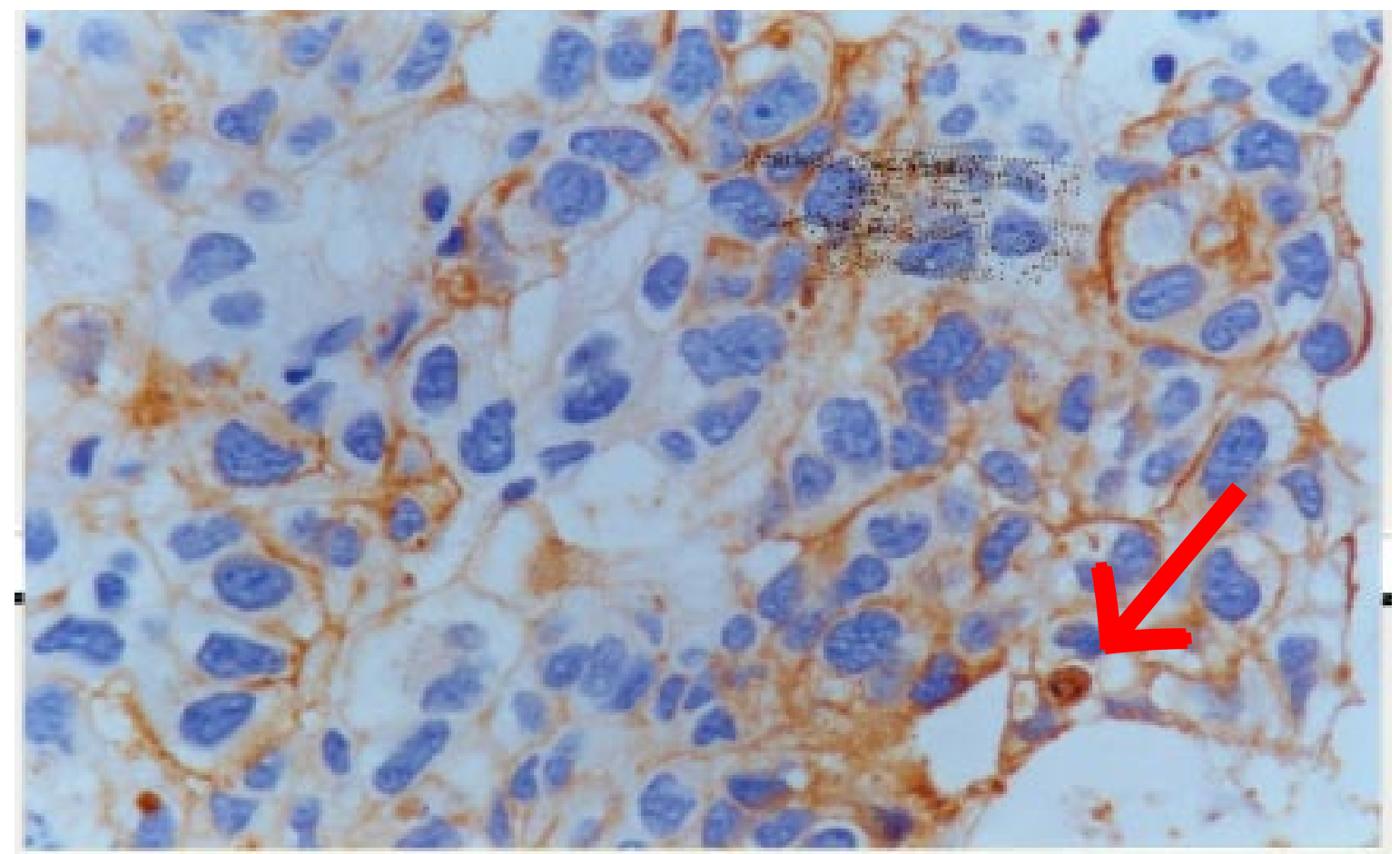

Foto 2. Alfa fetoproteina policlonal que marca citoplasma y glóbulos intracitoplasmáticos (cuerpos hialinos) (flecha). 
la cara posterior de la vejiga. No se observan signos de hidronefrosis. El resto de órganos abdominales, retroperitoneales y pélvicos son de caracteres normales.

Resonancia magnética: Proceso neoformativo intraraquideo y extradural, que mide $3.4 \mathrm{~cm}$ de diámetro y se localiza en el lado derecho del espacio epidural comprendido entre D7 y D11. Signos de compromiso secundario múltiple del hígado y ambos pulmones. Proceso neoformativo pélvico de localización retrovesical.

Examen físico:

Abdomen que a la palpación evidencia tumoración hipogastrica suprapubica dolorosa de 7 x $4 \mathrm{~cm}$.

Se evidencia a través del canal vaginal tejido rojo vinoso nodular aparentemente tumoral. Se explora con especulo virginal identificándose tumor que parece depender del utero y que infiltra el tercio superior de vagina procediendo a tomar biopsia.

Informe anatomopatológico 048949, Tumor del seno endodermal.

Inmunohistoquímica: Alfa fetoproteina (positiva), queratina (positiva) y PLAP (positivo).

20.06.02. Inicia terapia con Platino, Bleomicina y Etoposido.

\section{CASO II.}

Paciente de un año y dos meses de edad, natural y procedente de Lima, quien ingresa al INEN el 06.09.2002, con un tiempo de enfermedad de 15 días, referida del Instituto Nacional de Salud del Niño con el diagnóstico de sarcoma botroide. Desde su ingreso cursa con sangrado genital.

Examenes auxiliares: Hb (92), Hto (29.2), AFP $14970.0 \mathrm{ng} / \mathrm{ml}$. Los demás exámenes se encuentran dentro de los valores normales. Ecografía: Hígado, bazo, páncreas aparentemente normales. Se evidencia proceso expansivo heterogéneo, multilobulado en canal vaginal de 40 x 42 × $36 \mathrm{~mm}$. Radiografía de torax normal.

Estudio de medula ósea normal (10.09.2002).

Examen físico:

Abdomen blando, depresible, no signos peritoneales, RHA (+), no se palpan masas ni tumoraciones.

Genitales externos congestivos, lesión tumoral rojo vinosa sangrante en tercio inferior y medio de la vagina, difícil de resecar por la ubicación y dificultad técnica.

Se toma biopsia en sacabocado.

Informe de lámina AP.53252, Neoplasia maligna que sugiere tumor del seno endodermal.

Inmunohistoquímica: Alfa fetoproteina (positiva), desmina (negativa) y HCG (negativa).

18.09.2002. Pediatria: Inicia terapia con Platino, Bleomicina, Etoposido.

\section{DISCUSION}

La localización pélvica extragonadal del tumor del seno endodermal es rara si se excluyen las localizaciones presacra y sacrococcigea (4), de estos tumores en 30 a $40 \%$ de los casos la localización no es definida claramente, 10 a $15 \%$ se originan en cervix y $50 \%$ en vagina (3). El tumor del senoendodermal de localización vaginal y cervical se presenta como una masa sesil o polipoide, friable, blanco grisacea que protruye a través de la vagina y que incluye una historia de flujo vaginal sanguinolento $(1,3,4)$. Cuando se localiza en la vagina generalmente se origina en la pared vaginal posterior $(7,11)$ y el diagnóstico diferencial incluye al sarcoma botroides y el carcinoma de células claras $(3,4,6,11)$.

La neoplasia se caracteriza histológicamente por los cuerpos de Schiller Duval, estructuras glomeruloides constituidas por canales anastomoticos y tubulos tapizados por células cuboidales de citoplasma claro y núcleo hipercrómico $(3,12)$. La presencia de glóbulos hialinos PAS positivos, de localización intracelular y extracelular es esencial para el diagnóstico. A la microscopia electrónica se identifican como material electrodenso y a la inmunohistoquímica marcan intensamente para alfa fetoproteina $(6,9)$.

La neoplasia se presenta casi exclusivamente en niñas menores de tres años $(1,4,10,11,12)$. En nuestra revisión encontramos un reporte de una niña de 10 años con diagnóstico de tumor del seno endodermal localizado en la pared posterior de la vagina (6).

La inmunohistoquímica con alfa fetoproteina es positiva en casi todos los casos y es útil para el diagnostico diferencial. También es positiva para alfa 1 antitripsina, CD15, albumina y citoqueratina $(3,11,12)$.

La media de supervivencia es de 11 meses y 10 a $15 \%$ mueren dentro de los 2 años. De 35 casos con seguimiento clínico solo tres han reportado 


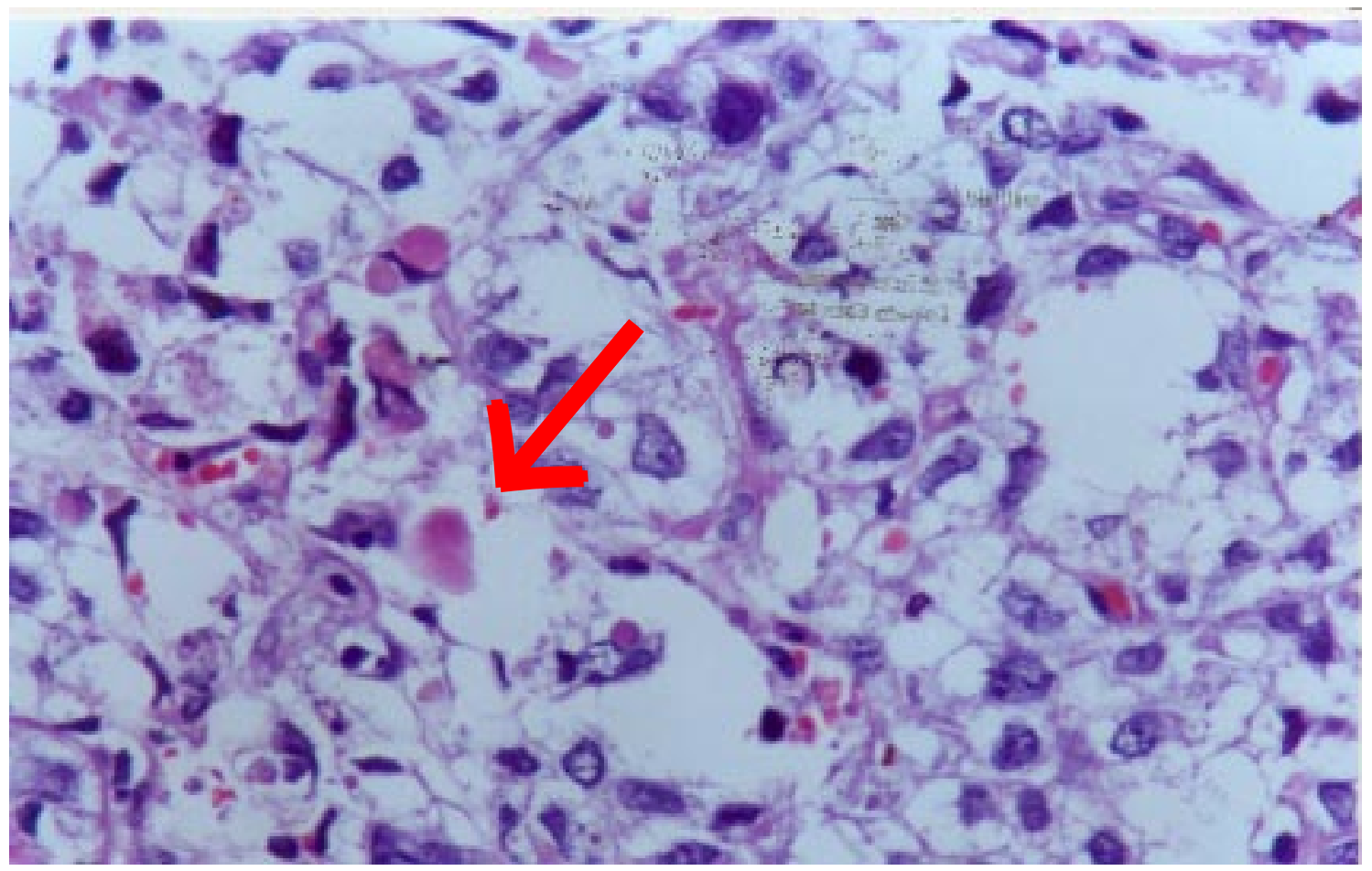

Foto 3. Tumor del seno endodermal que evidencia la presencia de cuerpos hialinos. H\&E x 400 .

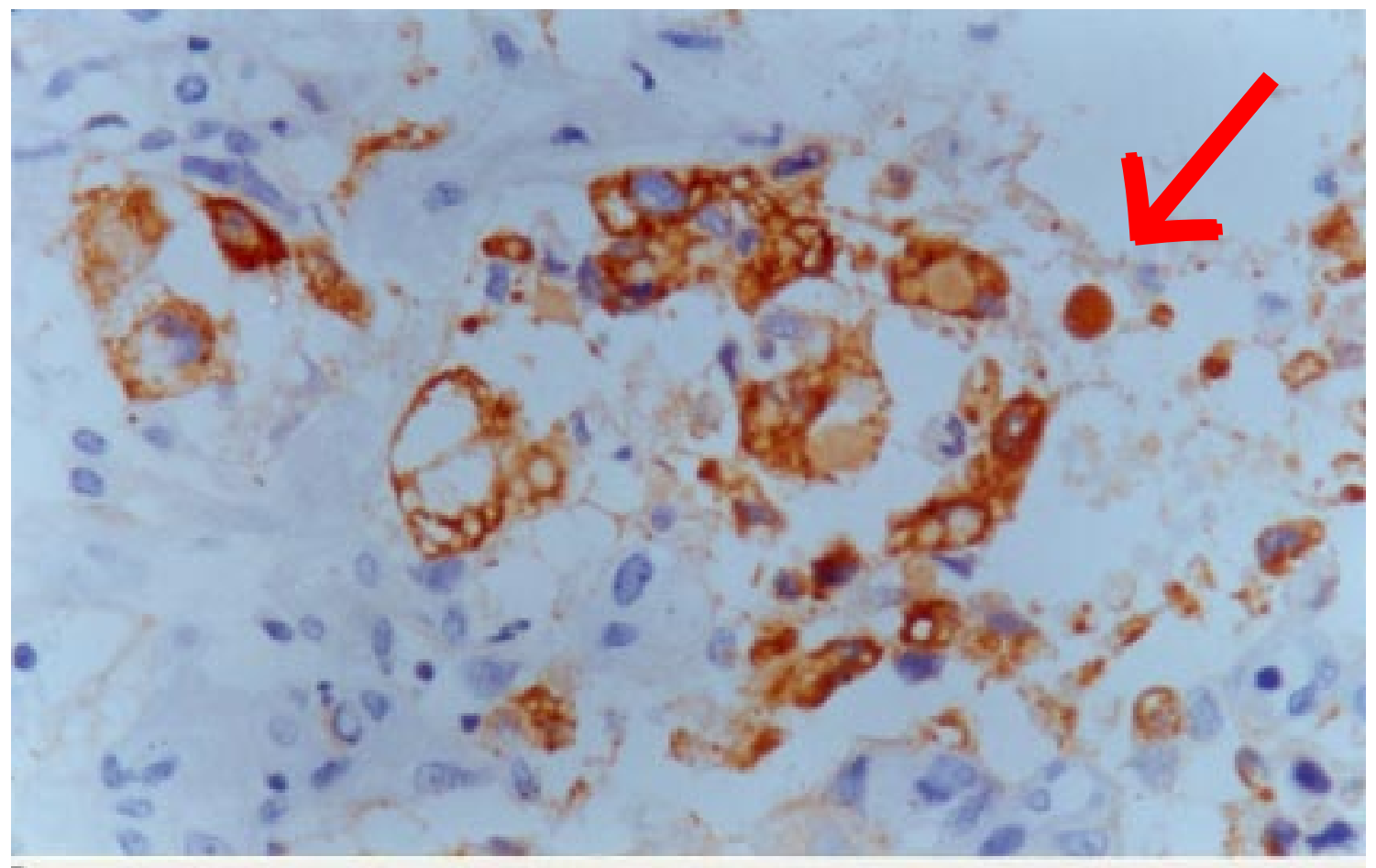

Foto 4. Inmunohistoquímica que marca positivo para alfa fetoproteina a los glóbulos hialinos. H\&E x 400. 
supervivencia a 5 años (3). El tumor no responde a la radioterapia, pero el tratamiento combinado de quimioterapia y cirugía ha mejorado considerablemente el pronóstico. El tratamiento con VAC (Vincristina, Dactinomicina y Ciclofosfamida) se describe como el tratamiento de elección, produciendo reducción del volumen local del tumor (5).

\section{Correspondencia:}

Dr. Juvenal Sanchez Lihon.

Jefe del Departamento de Patología.

Instituto Nacional de Enfermedades Neoplásicas.

Av. Angamos 2520. Surquillo - Lima.

Telefonos: 4499137 - 2171300.

\section{REFERENCIAS BIBLIOGRAFICAS}

1. Kurman R, Norris H, Wilkinson E. Tumors of the Vagina; Atlas of tumor Pathology Tumors of the Cervix Vagina and Vulva. Third Series Fascicle Four. Armed Forces Institute of Pathology, Bethesda Maryland 1992: 141 178.

2. Chong S, Wee A, Yeoh S, Nilsson B, Chan S. Retroperitoneal Endodermal Sinus Tumor Report of a Case With an Abnormal Cervicovaginal Smear. Acta Cytol 1994; 38: $562-567$.

3. Copeland L, Sneigge N, Ordoñez N, Hancock $\mathrm{K}, \mathrm{Gershenson} \mathrm{D}$. Endodermal Sinus tumor of the vagina and cervix. Cancer 1985; 55: 2558 - 2565.
4. Clement P, Young R, Scully R. Extraovarian Pelvic Yolk Sac Tumors. Cancer 1988; 62: 620 - 626.

5. Andersen W, Sabio H, Durso N, Mills S, Levien M. Endodermal Sinus Tumor of the Vagina. Cancer 1985; 56 : 1025 - 1027.

6. Ishi K, Suzuki F, Saito A, Koyatsu J, Kubota T. Cytodiagnosis of Vaginal Endodermal Sinus Tumor A Case Report. Acta Cytol 1998; 42: 399 - 402.

7. Imai A, Furui T, Yocoyama Y, Sawairi M, Shimokawa K. Case Report Endodermal Sinus Tumor of the Vagina in an Infant: Magnetic Resonance Imaging Evaluation. Gynecologic Oncology 1993; 48: 402 - 405.

8. Nogales F, Bergeron C, Carvia R, Alvaro T, Fulwood H. Ovarian Endometrioid Tumors with Yolk Sac Tumor Component an Unusual Form of Ovarian Neoplasm. Am J Surg Pathol 1996; 20 (9): 1056 - 1066.

9. Nogales F, Silverberg S, Bloustein P, Martínez-Hernandez A, Pierce B. Yolk Sac Carcinoma (Endodermal Sinus Tumor) Ultrastructure and Histogenesis of Gonadal and Extragonadal Tumors in Comparison with Normal Human Yolk Sac. Cancer 1977; 39: 1462 - 1474.

10.Parham D, Bugg M, Pratt C. Carcinomas, Adenomas, Precursor Lesions and Second malignancies; Pediatric neoplasia Morphology and Biology. Lippincott - Raven Publishers 1996: 363 - 404

11.Rosai J. Female Reproductive System; Ackerman's Surgical Pathology. Eight Edition, Mosby 1996: 1319 1352.

12.Spatz A, Bouron D, Pautier P, Castaigne D, Duvillard P. Case Report Primary Yolk Sac tumor of the Endometrium: A Case Report and Review of the Literature. Gynecologic Oncology 1998; 70: 285 - 288. 